Herz 2020 · 45 (Suppl 1):S78-S79

https://doi.org/10.1007/s00059-019-4821-8

Published online: 28 May 2019

(c) Springer Medizin Verlag $\mathrm{GmbH}$, ein Teil von Springer Nature 2019

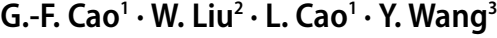

'Department of Neurology, Beijing Anzhen Hospital, CapitalMedical University, Beijing, China ${ }^{2}$ Center For Medical Device Evaluation, NationalMedical Products Administration, Beijing, China ${ }^{3}$ Department of Pathology, Sir Run Run Shaw Hospital, Zhejiang University School of Medicine, Zhejiang, China

\section{Erratum to: Stroke in patients with prosthetic valve endocarditis}

\section{Single-center cohort study in China}

\section{Erratum to:}

\section{Herz 2019}

https://doi.org/10.1007/s00059-019-

4809-4

The original article has been corrected. The data given in - Table 4 for patient No. 13 were wrong. Please find here the corrected Table.

The authors and publisher apologize for this mistake.

\section{Corresponding address}

\section{G.-F. Cao}

Department of Neurology, Beijing Anzhen Hospital, CapitalMedical University

NO 2 Anzhen Road, 100029 Beijing, China caoguifang2013@sina.cn 


\begin{tabular}{|c|c|c|c|c|c|c|c|c|c|c|}
\hline No & Sex & Age & Causes & $\begin{array}{l}\text { Mechanical } \\
\text { valve }\end{array}$ & $\begin{array}{l}\text { Location of } \\
\text { vegetations }\end{array}$ & $\begin{array}{l}\text { Type of } \\
\text { stroke }\end{array}$ & $\begin{array}{l}\text { Nervous system } \\
\text { manifestations }\end{array}$ & $\begin{array}{l}\text { Vascular } \\
\text { distribution }\end{array}$ & Blood culture & Death \\
\hline 1 & $\mathrm{~F}$ & 51 & RHD & Yes & MV & $\mathrm{SAH}$ & Headache, coma & Unknown & Negative & Yes \\
\hline 2 & $\mathrm{~F}$ & 47 & RHD & Yes & MV & IS & Left hemiplegia & Both ICA & Negative & No \\
\hline 3 & M & 23 & RHD & Yes & AV & IS & $\begin{array}{l}\text { Right hemiplegia, } \\
\text { dysarthria }\end{array}$ & Left ICA & Negative & No \\
\hline 4 & M & 24 & RHD & Yes & $\mathrm{AV}$ & $\mathrm{ICH}$ & $\begin{array}{l}\text { Headache, coma, } \\
\text { left hemiplegia }\end{array}$ & Right ICA & Negative & Yes \\
\hline 5 & M & 54 & RHD & Yes & MV & Unknown & Coma, vomiting & Unknown & Negative & Yes \\
\hline 6 & M & 55 & RHD & Yes & MV & IS & Dizziness & Left ICA, VBA & No & No \\
\hline 7 & M & 64 & RHD & Yes & LVOT & Unknown & $\begin{array}{l}\text { Coma, right hemi- } \\
\text { plegia }\end{array}$ & Unknown & No & Yes \\
\hline 8 & M & 42 & RHD & Yes & MV & $\mathrm{ICH}$ & Coma, vomiting & Left ICA & Candida glabrata & Yes \\
\hline 9 & M & 79 & IE & No & $\mathrm{MV}, \mathrm{AV}$ & IS & Dizziness & $\begin{array}{l}\text { Right ICA, } \\
\text { VBA }\end{array}$ & Enterococci & No \\
\hline 10 & M & 73 & $\mathrm{AS}$ and $\mathrm{Al}$ & No & $\mathrm{AV}$ & IS & Dysarthria & Left ICA, VBA & Candida albicans & No \\
\hline 11 & M & 60 & Al & Yes & AV & IS & $\begin{array}{l}\text { Left hemiplegia, } \\
\text { dysarthria }\end{array}$ & Right ICA & $\begin{array}{l}\text { Streptococcus } \\
\text { gustis }\end{array}$ & No \\
\hline 12 & $\mathrm{~F}$ & 44 & RHD & Yes & MV & IS & $\begin{array}{l}\text { Dizziness, right } \\
\text { hemiplegia }\end{array}$ & Left ICA VBA & Enterococci & No \\
\hline 13 & M & 63 & IE & No & AV & IS & $\begin{array}{l}\text { Right hemiplegia, } \\
\text { aphasia }\end{array}$ & Left ICA & $\begin{array}{l}\text { Candida parap- } \\
\text { silosis }\end{array}$ & No \\
\hline 14 & $\mathrm{~F}$ & 76 & RHD & No & MV & IS & $\begin{array}{l}\text { Right hemiplegia, } \\
\text { lethargy }\end{array}$ & Both ICA & S. epidermidis & No \\
\hline 15 & M & 51 & DAR & Yes & AV & IS & Left hemiplegia & Right ICA & $\begin{array}{l}\text { Candida parap- } \\
\text { silosis }\end{array}$ & No \\
\hline 16 & $M$ & 67 & BAV & Yes & $\mathrm{MV}, \mathrm{AV}$ & IS & Left hemiplegia & Right ICA & S. epidermidis & No \\
\hline 17 & M & 60 & RHD & Yes & MV & IS & Syncope & Both ICA & S. aureus & No \\
\hline 18 & M & 66 & NS & NO & AV & ICH & Headache, seizure & VBA & S. aureus & No \\
\hline
\end{tabular}

